\title{
Optical trapping and sensing with plasmonic dipole antennas
}

\author{
Weihua Zhang and Olivier J. F. Martin \\ Nanophotonics and Metrology Laboratory, Swiss Federal Institute of Technology Lausanne (EPFL), \\ 1015 Lausanne, Switzerlan
}

\begin{abstract}
In this work, we study how to use a plasmonic dipole antenna as a multifunctional nanodevice for surface-enhanced Raman spectroscopy (SERS), localized surface plasmon resonance (LSPR) -based sensing and optical trapping. An analytical model is implemented to link the local electric field enhancement with the gradient forces, as well as the resonance shift caused by the presence of the analyte which can be a molecule or a nanoparticle. We find that a higher local field enhancement induces stronger trapping forces and a larger resonance wavelength shift. Experiments were also performed using plasmonic dipole antennas. Strong SERS signals were observed from the nanogap of an antenna, trapping of Au nanoparticles as small as $10 \mathrm{~nm}$ was achieved with a moderate laser power, and evident resonance shifts of the antenna associated with the trapping events were also observed. These results are consistent with our theoretical result that the giant field enhancement generated by a plasmonic dipole antenna also generates strong gradient forces and a high spectral sensitivity.
\end{abstract}

Keywords: plasmonic dipole antenna, surface-enhanced Raman spectroscopy, optical trapping, sensing

\section{INTRODUCTION}

Driven by rapid developments in the field of microfluidics, the idea of integrating miniaturized functional elements into a small device (i.e., the lab-on-a-chip) has attracted a lot of interests because of its great application potential in future markets. ${ }^{1}$ As a result, many endeavors have been undertaken to discover the nanometer equivalent of conventional functional elements. Among all these efforts, the research on plasmonic-based techniques is particularly active because of the superb capabilities of plasmonic structures in many important fields, such as sensing, spectroscopy and optical manipulation, which represent the most important tools in modern chemical and biological analyses. ${ }^{2-4}$ In this work, we focus on one special group of plasmonic structures - plasmonic antennas - which are also known as optical antennas since they work in the optical regime. ${ }^{5,6}$

Plasmonic antennas are metal nanostructures which can effectively link near-field signals with propagating waves in the far-field zone. In this sense, they can be treated as the counterpart of conventional antennas for radio frequency, although, due to the excitation of the localized surface plasmon resonances, plasmonic antennas have a much higher farfield vs. near-field conversion efficiency than conventional antennas. ${ }^{5,7}$ Moreover, different from traditional antennas whose size is comparable or larger than their operating wavelength, a plasmonic antenna can be much smaller than its operating wavelength. Simple scaling rules between plasmonic and traditional antennas can be established if the building blocks of the antenna are nanorods. ${ }^{8}$ This size effect is fundamentally important because it breaks the diffraction limit the rule rooted in the wave nature of light - and allows us to build efficient subwavelength optical devices. ${ }^{9}$

Thanks to these extraordinary properties, plasmonic antennas have been successfully implemented in many different fields to enhance the performance of traditional optical techniques. First, they represent a perfect signal transducer which can effectively transduce nano-events occurring in the near-field regime to signals in the far-field zone that can be recorded with traditional optical devices. ${ }^{10}$ Second, a plasmonic antenna can collect propagating light with a very high collection efficiency - it can exhibit a scattering cross section much larger than its geometrical size - and build up a very high field intensity at "hot spots" which correspond to the feeding position of a conventional antenna. ${ }^{11}$ Since the fields are strong and localized, these "hot spots" are also associated with a large gradient of the field intensity and consequently large gradient forces on nano-objects., 12,13

Inspired by specific applications, many studies have been performed in order to utilize different types of plasmonic antennas for different purposes. One line of these studies has focused on creating "hot spots" at specific desired

Plasmonics: Metallic Nanostructures and Their Optical Properties VIII, edited by Mark I. Stockman,

Proc. of SPIE Vol. 7757,775712 • @ 2010 SPIE · CCC code: 0277-786X/10/\$18 · doi: 10.1117/12.864225

Proc. of SPIE Vol. 7757 775712-1 
wavelengths, leading to many dfferent designs proposed and investigated in the last few years. Particularly, the plasmonic dipole antenna - a nanorod pair spaced by a nanometer gap - has been found an ideal candidate for this purpose, since its resonance frequency and field enhancement can be tailored by changing its length and gap size. ${ }^{6,11}$ Another line of studies are LSPR-based sensing, which was first developed by Van Duyne and his colleagues. ${ }^{4}{ }^{14}$ In analogy to conventional SPR sensors, chemical events (i.e., binding and dissociation) are detected in real-time by monitoring the resonance wavelength of a plasmonic antenna. The small size of the plasmonic antenna makes this technique a perfect candidate for the large scale integration, which is particularly important in the field of parallel sensing. ${ }^{2}$ Another trend of research is to trap nano-objects, even a single protein molecule, which is an extremely difficult task for conventional trapping techniques. For instance, Quidant and his group have successfully trapped single bacteria with plasmonic nanoantennas recently. ${ }^{12}$

In this work, we will first reveal the links between the field enhancement, optical forces and resonance shifts, using a theoretical model. Then we will demonstrate that all the functions described in the previous paragraph can be achieved by using the same plasmonic dipole antenna. That is to use the antenna to trap a particle to its vicinity and then detect it with spectroscopic techniques.

\section{LINKS BETWEEN SERS, RESONANCE SHIFT AND OPTICAL FORCES}

There are intrinsic links between the performances of plasmononic antennas for SERS, sensing and optical trapping, although these functions are based on the measurement of different physical quantities. The enhancement of the electric field governs the enhancement factor for SERS; resonance wavelength shifts are normally the value monitored in sensing experiments; the field intensity gradient determines the optical trapping forces. In the following paragraphs, we will use a simplified model to connect these different quantities.

Let us consider an isolated plasmonic antenna. For simplicity, an elliptical nanorod is chosen here to model the optical properties of the plasmonic antenna because its polarizability can be simply written as:

$$
\alpha=\frac{\varepsilon_{a}-\varepsilon_{l}}{\varepsilon_{l}+\left(\varepsilon_{a}-\varepsilon_{l}\right) L} \varepsilon_{0} V,
$$

where $V$ is the volume of the particle, $L$ is a parameter defined by the ratio between the two axes, $\varepsilon_{a}$ and $\varepsilon_{l}$ are the permittivity of the plasmonic antenna and surrounding liquid, respectively. Equation (1) is derived for elliptical nanoparticles, but it is generally correct for other antenna structures, for which $L$ is decided by the shape of the structure. ${ }^{15}$ When the dominator in Eq. (1) reaches its minimum, the resonance occurs. Hence, the resonance condition is

$$
\varepsilon_{a}\left(\lambda_{\text {res }}\right)=\frac{L-1}{L} \varepsilon_{l} .
$$

From the knowledge of the resonant properties of the antenna, the local field can be phenomenologically written in the form $E(r)=\alpha(\lambda) A(r) E_{0}$ where $E_{0}$ is the incident field, $\alpha$ includes the wavelength dependence, and $A(r)$ describes the geometry related factors. In other words, for a specific geometry, we can divide the electric field enhancement

$$
g_{E}=E(r) / E_{0}=\alpha(\lambda) A(r)
$$

into two parts, the resonant enhancement $\alpha(\lambda)$ and the geometrical enhancement $A(r)$.

When a molecule is located in the vicinity of this plasmonic antenna, its influence on the local field is normally negligeable since the polarizability of the antenna is much larger than that of the molecule. Considering that both the excitation and radiation of Raman scattering are enhanced by the antenna, the Raman enhancement is

$$
g_{R}=g_{E}{ }^{4} .
$$

The trapping forces on the molecule can also be enhanced in the vicinity of the antenna. Since the molecule is small compared to the wavelength, it will mainly experience gradient forces which push the molecule towards the spot with the highest local field enhancement, namely the "hot spot". Under the dipole approximation, the gradient force is

$$
F\left(r_{m}\right)=\operatorname{Re}\left(\alpha_{m}\right) \nabla\left|E_{a}\left(r_{m}\right)^{2}\right|,
$$

where $\alpha_{m}$ is the polarizability of the molecule and $E_{a}\left(r_{m}\right)$ the local electric field intensity generated by the antenna. ${ }^{9}$ 
The presence of an antenna strongly enhances the field gradient $\nabla\left|E_{a}\left(r_{m}\right)^{2}\right|$. This enhancement not only comes from the intensity enhancement but also from the strong localization of the electric field in the vicinity of the antenna. ${ }^{5}$ In a typical case, the near-field components drop to almost zero within a few tens of nanometers, one order of magnitude smaller than the wavelength. Therefore, the trapping forces can be enhanced by one order of magnitude, only thanks to the localization of the electric field.

Although the response of the system is dominated by the plasmonic antenna, the presence of the molecule can also modulate its resonance wavelength. This allows us to detect the chemical binding events in the vicinity of the plasmonic antenna by monitoring its resonance wavelength. In the following part, we will seek the link between the resonance shift and the parameters $\alpha_{m}$ and $g_{E}$.

In order to investigate this resonance shift, the coupling between the molecule and the antenna needs to be considered. We assume that the size of the plasmonic antenna is much smaller than the wavelength. Hence, the coupling between these two nanoobjects can be described by a pair of coupled dipole.

$$
\begin{aligned}
& P_{a}=\alpha_{a}\left(E_{0}+E_{m}\right) \\
& P_{m}=\alpha_{m}\left(E_{0}+E_{a}\left(r_{m}\right)\right)
\end{aligned}
$$

where $E_{0}$ is the incident electric field, $E_{\mathrm{m}}$ and $E_{\mathrm{a}}$ are secondary fields (i.e., the scattered fields), $P_{\mathrm{a}}$ and $P_{\mathrm{m}}$ are the dipole moments, and the subscripts ' $a$ ' and ' $m$ ' denote the antenna and the perturbing molecule (or nanoparticle), respectively. As mentioned previously, the secondary fields can be written as

$$
\begin{aligned}
& E_{a}(r)=A_{a}\left(r_{m}\right) P_{a}(\lambda) \\
& E_{m}(r)=A_{m}\left(r_{a}\right) P_{m}(\lambda)
\end{aligned}
$$

Substituting $E_{\mathrm{p}}$ and $E_{\mathrm{m}}$ in Eq. (6) with Eq (7), the dipole moment of the antenna becomes

$$
P_{a}=\frac{\alpha_{a}+A_{m}\left(r_{m}\right) \alpha_{m}}{1-A_{m}\left(r_{a}\right) A_{a}\left(r_{m}\right) \alpha_{a} \alpha_{m}} E_{0} .
$$

The resonance of the antenna is determined by the dominator of Eq. (8). The resonance occurs when the dominator of reaches its minimum:

Inserting Eq (1) in Eq (9), we obtain

$$
1-A_{a}\left(r_{m}\right) A_{a}\left(r_{m}\right) \alpha_{a} \alpha_{m}=0
$$

$$
\varepsilon_{a}\left(\lambda_{\text {res }}\right)=\frac{L-1-A_{a} A_{m} \alpha_{m} V}{L-A_{a} A_{m} \alpha_{m} V} \varepsilon_{l} .
$$

Considering that the impact of the molecule on the antenna is small, Eq. (10) can then be rewritten as

$$
\varepsilon_{a}\left(\lambda_{\text {res }}\right)=\varepsilon_{a}\left(\lambda_{\text {res } 0}\right)-\Delta / L^{2}
$$

where $\Delta=A_{a} A_{m} \alpha_{m} V \varepsilon_{l}$ and $\lambda_{\text {res } 0}$ is the resonance wavelength of the unperturbed antenna, which is determined by Eq (2). Hence, the resonance wavelength shift is

$$
\delta \lambda=\left(\Delta / L^{2}\right) d \lambda /\left.d \varepsilon\right|_{\lambda 0}=\left(A_{a} A_{m} \alpha_{m} V \varepsilon / L^{2}\right) d \lambda /\left.d \varepsilon\right|_{\lambda 0} .
$$

It is interesting that the shift is not directly related to $\alpha_{a}$ which contains the resonance effects of the antenna. Since $A_{a}$ is the geometry factor in the electric field enhancement, Eq. (12) tells us that the resonance wavelength shift (i.e., the spectral sensitivity) is determined by the geometry induced field enhancement of the plasmonic structure.

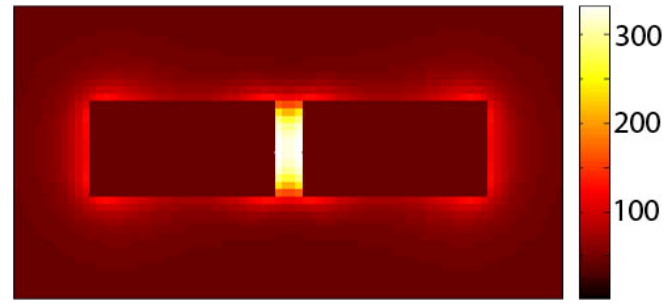

Figure 1: Field intensity map of a plasmonic dipole antenna excited by a plane wave polarized along its axis. 
Although an elliptical nanoparticle has been used to model the plasmonic antenna, this result is expected to hold for other structures, since the above model is based on the coupled dipole approximation which is generally correct when the particle is much smaller than the wavelength.

It has been reported that a plasmonic dipole antenna is capable of generating a giant field enhancement in its nanogap. ${ }^{6}$ To demonstrate this field enhancement effect, we implemented the Green's tensor technique to simulate light scattering by the dipole antenna, as shown in Fig 1. An enhancement of the field intensity in excess of 300 times is observed in the nanogap. Therefore, an antenna which generates a good field enhancement can be utilized as a good SERS substrate, sensitive sensor ${ }^{10}$ and a high performance trapping device.

\section{EXPERIMENTAL}

Let us now verify the above theory experimentally. Two-dimensional Au dipole antenna arrays were fabricated with electron beam lithography. ${ }^{11}$ Figure 2 shows the SEM image of a typical antenna. The height and width of the antennas were $40 \mathrm{~nm}$; the length and gap size of the antennas were varied over the arrays. The distance between two neighboring antennas was $3 \mu \mathrm{m}$, much larger than the wavelength, so that the optical response from single antennas could be measured without being influenced by neighboring structures.

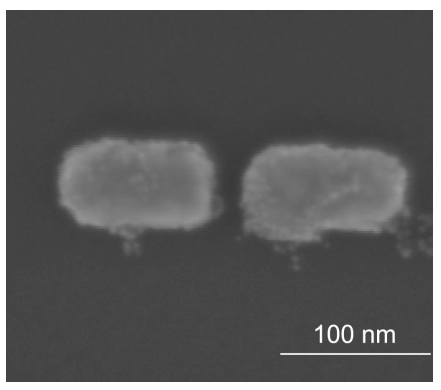

Figure 2: SEM image of a typical nanofabricated plasmonic dipole antenna.

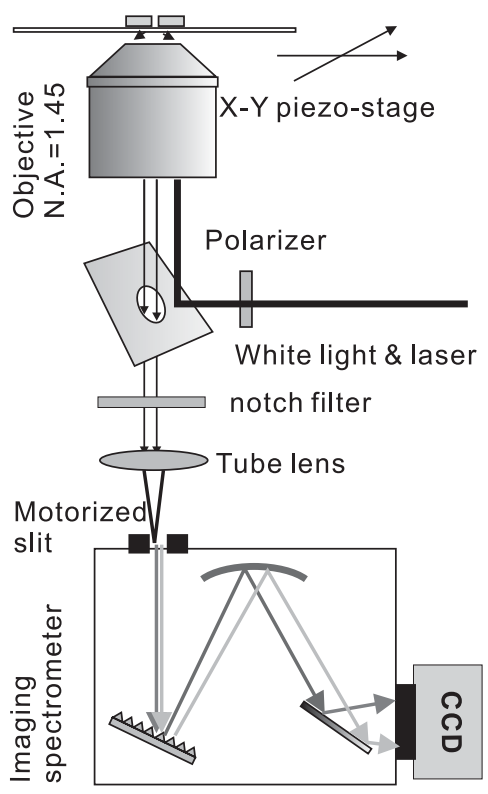

Figure 3: Optical setup used in this work; it is a combination of a inverted microscope and an imaging spectrograph. 
The experiments were performed on an inverted optical microscope (Olympus IX-71). The trapping and excitation lasers, as well as the white light beam for characterizing the LSPRs of the antennas, were injected from the back port of the microscope. A partially coated glass was used as a mask to perform a dark-field type of measurement. The light beams were reflected from the peripheral area of the mask, subsequently went through the edge of the objective, and generated an evanescent wave at the sample/air interface to excite the antennas. The direct reflection of the incident field was blocked by the mask, so that only the scattered fields entered the spectrograph and were recorded by the CCD camera.

\section{RESULT AND DISCUSSION}

We first tested the performance of the antennas as SERS substrate. Benzenethiol molecules were used as analytes in the measurement. Thanks to the Au-S bond, benzenethiol molecules formed a submonolayer on the antenna. Considering the size of the antenna, we can estimate that there were less than 100 molecules in one active spot, i.e., in the nanogap of an antenna. After being excited by a $1 \mathrm{~mW}$ HeNe laser, strong Raman peaks were observed. It is known that benzenethoil does not show resonance Raman effects in the visible range and consequently has a very small Raman scattering cross section, ${ }^{11}$ so the huge field enhancement generated by the antenna gap is demonstrated.



Figure 4: Surface-enhanced Raman spectrum of benzenthiol molecules adsorbed on a single plasmonic dipole antenna. The collection time was $1 \mathrm{~s}$.

Trapping and sensing experiments were also performed with the same sample. To this end, a trapping laser and white light beam were used simultaneously for trapping nanoparticles and monitoring the resonance wavelength of the antennas, respectively. ${ }^{13} \mathrm{Au}$ nanoparticles were used to demonstrate the strong trapping forces in the "hot spots". The measurements were performed in the following way.The antennas were first measured in deionized water to ensure that the system was stable. Then, $20 \mathrm{~nm}$ Au colloidal (Sigma-Aldrich) was added into the solution and the scattering spectra of the antennas were collected at a repetition rate of 1 frame per second. Finally, the trapping laser was turned off and scattering spectra of the antennas were collected further.

Figure 5 shows one typical result. The resonant wavelength of the antenna remained constant until Au nanopartices were trapped in the gap, leading to a stepwise shift of the antenna resonance frequency. This illustrated how to use a plasmonic dipole antenna for both trapping and sensing nanoobjects.

The trapping potential generated by the antenna can be estimated using numerical techniques. For a $10 \mathrm{~nm} \mathrm{Au}$ nanoparticle, a trapping potential several times lager than the kinetic energy of Brownian motions can be obtained, with an incident illumination power as low as $2 \mathrm{~mW} / \mathrm{mm}^{2}$, this is 3 orders of magnitude larger than the forces provided by a conventional trapping setup with the same illumination.

In addition, the resonance shift generated by the Au nanoparticle is about $5 \mathrm{~nm}$, which is far beyond the detection limit which is estimated to be $\sim 0.3 \mathrm{~nm}$. Therefore, single molecule sensitivity can be expected for this plasmonic dipole antenna based sensor. 

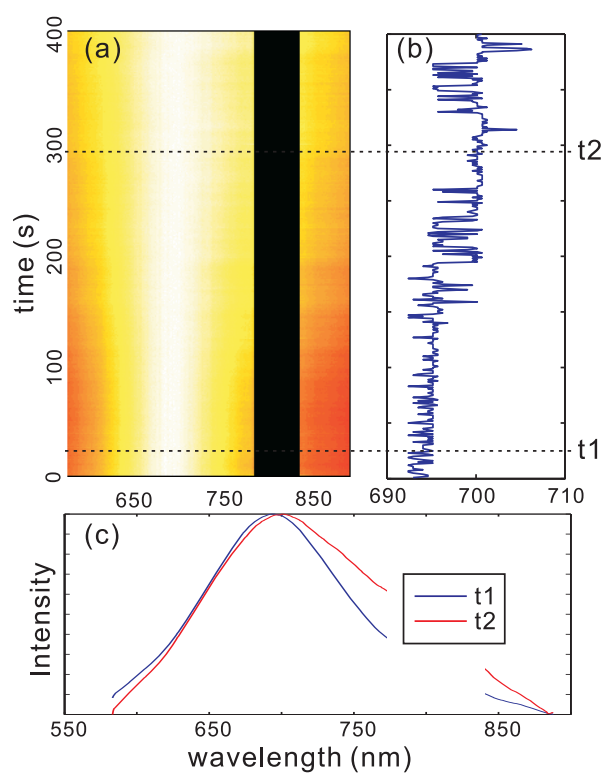

Figure 5: Experimental results for a typical trapping experiment. (a) Waterfall plot of the scattering spectra of a plasmonic antennas as a function of time. (b) Time trace of the peak wavelength of the spectra in (a). (c) Spectra snapshot at $\mathrm{t} 1$ and $\mathrm{t} 2$.

An interesting phenomenon in Fig. 5 is that the resonance is broadened when a particle becomes trapped. There are two possible reasons for this: (1) once an Au particle is trapped, the total radiation loss increases, or (2) the trapped Au nanoparticle increases the Ohmic losses of the system.

\section{SUMMARY}

In summary, we have studied the intrinsic links between the electric field enhancement, the optical forces and the resonance shifts for plasmonic antennas. Our results indicate that the nanogap of a plasmonic dipole antenna provides a nanoscale multifunctional workbench for manipulating, detecting and analyzing nano-objects. Motivated by this idea, proof-of-principle experiments were performed. We have successfully demonstrated that nanofabricated plasmonic dipole antennas are tremendous SERS substrates, have a superb spectral sensitivity, and can be used for trapping particles only a few nanometer in size.

Acknowledgements: This work was supported by the EPFL-STI seed fund and the Swiss National Science Foundation (Grant 200021-113735)

\section{REFERENCES}

[1]. LI, P. C. H., [Microfluidic Lab-on-a-Chip for Chemical and Biological Analysis and Discovery] Taylor \& Francis Group, Boca Raton \& London New York, (2005).

[2] Stewart, M. E., Anderton, C. R., Thompson, L. B., Maria, J.; Gray, S. K., Rogers, J. A., Nuzzo, R. G., "Nanostructured plasmonic sensors," Chem. Rev. 108 (2), 494-521 (2008).

[3] Quidant, R., Girard, C., "Surface-plasmon-based optical manipulation," Laser \& Photonics Rev. 2, 47-57 (2008).

[4] Willets, K. A.; Van Duyne, R. P., "Localized surface plasmon resonance spectroscopy and sensing," Annu. Rev. Phys. Chem. 58, 267-297 (2007).

[5] Muhlschlegel, P., Eisler, H. J., Martin, O. J. F., Hecht, B., Pohl, D. W., "Resonant optical antennas," Science 308 (5728), 1607-1609 (2005). 
[6] Fischer, H., Martin, O. J. F., "Engineering the optical response of plasmonic nanoantennas," Opt. Express 16 (12), 9144-9154 (2008).

[7] Kreibig, U., Vollmer, M., [Optical properties of Metal Clusters], Springer-Verlag, Berlin Heidelberg, (1995).

[8] Novotny, L., "Effective wavelength scaling for optical antennas," Phys. Rev. Lett. 98 (26), 266802 (2007)

[9] Novotny, L., Hecht, B., [Principles of Nano-Optics]. Cambridge University Press, New York, (2006).

[10] Alu, A., Engheta, N., "Tuning the scattering response of optical nanoantennas with nanocircuit loads," Nat. Photonics 2 (5), 307-310 (2008).

[11]Zhang, W. H., Fischer, H., Schmid, T., Zenobi, R., Martin, O. J. F., "Mode-Selective Surface-Enhanced Raman Spectroscopy Using Nanofabricated Plasmonic Dipole Antennas," J. Phys. Chem. C 113 (33), 14672-14675 (2009).

[12] Righini, M., Volpe, G., Girard, C., Petrov, D., Quidant, R., "Surface plasmon optical tweezers: Tunable optical manipulation in the femtonewton range," Phys. Rev. Lett. 100 (18), 186804 (2008)

[13]Zhang, W. H., Huang, L. N., Santschi, C., Martin, O. J. F., "Trapping and sensing $10 \mathrm{~nm}$ metal nanoparticles using plasmonic dipole antennas," Nano Lett. 10, 1006 (2010).

[14]Haes, A. J., Van Duyne, R. P., "A nanoscale optical blosensor: Sensitivity and selectivity of an approach based on the localized surface plasmon resonance spectroscopy of triangular silver nanoparticles," J. Am. Chem. Soc. 124 (35), 10596-10604 (2002).

[15] Myroshnychenko, V.; Rodriguez-Fernandez, J.; Pastoriza-Santos, I.; Funston, A. M.; Novo, C.; Mulvaney, P.; Liz-Marzan, L. M.; de Abajo, F. J. G., "Modelling the optical response of gold nanoparticles," Chem. Soc. Rev. 37 (9), 1792-1805 (2008) 